\author{
Karim Asehnoune \\ Richard S. Hotchkiss \\ Guillaume Monneret
}

\section{Understanding why clinicians should care about danger-associated molecular patterns}

Received: 17 December 2015

Accepted: 17 December 2015

Published online: 24 February 2016

(C) Springer-Verlag Berlin Heidelberg and ESICM 2016

K. Asehnoune $(\bowtie)$

Department of Anesthesiology and Critical Care Medicine, Hôtel Dieu, Centre hospitalier universitaire (CHU) de Nantes, 1 Place

Alexis Ricordeau, 44000 Nantes, France

e-mail: Karim.Asehnoune@chu-nantes.fr

\section{R. S. Hotchkiss}

Departments of Anesthesiology, Medicine, and Surgery,

Washington University School of Medicine, St Louis, MO, USA

\section{G. Monneret}

Immunology Department, Hôpital Edouard Herriot , Hospices Civils de Lyon, 69003 Lyon, France

In their recent article in Intensive Care Medicine, Timmermans et al. [1] report on plasma levels of dangerassociated molecular patterns (DAMPs) associated with immunosuppression in trauma patients. Over a 10-day monitoring period (starting at the trauma scene), the authors measured a number of parameters in 166 adult patients, including several plasma DAMPs (i.e. mitochondrial DNA, nuclear DNA and heat shock protein-70), cytokines and HLA-DR (human leukocyte antigen-antigen D related) mRNA, and performed functional testing (whole blood cytokine release upon lipopolysaccharide challenge), which is considered to be a good marker of innate immunity functionality. This study provides insight on several aspects of trauma-induced immunosuppression.

A major surprising finding was the rapidity with which immunosuppression occurs in trauma patients. Indeed, by comparing blood samples collected at the trauma scene with those collected at the emergency room, the investigators showed that HLA-DR mRNA was already significantly reduced by the time the patient arrived at the emergency room, accompanied by increased levels of the immunosuppressive cytokine interleukin-10 (IL-10). Other findings showed increased levels of circulating IL- 6 but unchanged levels of tumor necrosis factor alpha (TNF- $\alpha)$. Increased levels of circulating DAMPs occurred in parallel with the changes in the levels of these cytokines. Most importantly, results from whole blood LPS stimulation studies showed an increased release of IL-10 and a decreased release of IL- 6 and TNF- $\alpha$ in a subgroup of patients. All of these parameters (including functional testing) are established features of injury-induced immunosuppression [2,3]. This finding is particularly important because it contrasts sharply with the prevailing theory of immune dysfunction following trauma and with the results of a recent study in trauma patients which examined gene expression in circulating whole blood [4]. This latter study showed that trauma patients had downregulation of genes controlling adaptive immunity but upregulation of genes controlling innate immunity. On this basis, a number of investigators have hypothesized that the host immune response after trauma is a state of protracted, unabated inflammation driven by the innate immune system inducing organ dysfunction. In contrast, based on the findings of Timmermans et al. [1], it appears that immediately following the trauma, even at the trauma scene, circulating innate cells are already altered or unresponsive despite a potential upregulation of non-tolerizable genes (not measured by the authors). Obviously, distinct compartmentalized effects in organs cannot be ruled out but, at least in circulation, immunosuppression would appear to be the dominating force and that this force includes innate immunity.

Also in support of the hypothesis that immunosuppression is a key early driving force after trauma is the observation of the authors that an HLA-DR mRNA ratio (between day 3 and emergency room samples) of $<1$ was 
associated with an increased rate of secondary infections. This finding confirms, at the transcriptional level, protein results initially obtained by flow cytometry [5]. In their recently published study in Intensive Care Medicine, Gouel-Chéron et al. [6] showed that monocyte HLA-DR (mHLA-DR) nadir occurs at day 2 after trauma. Those patients for whom the mHLA-DR levels are not restored within the following days are at increased risk for infections $[5,6]$. Another intriguing finding of Timmermans et al. [1] is the demonstration that HLA-DR mRNA expression determined in the emergency room negatively correlates with the levels of DAMPs. This correlation agrees with previously reported results showing a negative correlation between mHLA-DR and IL-6 measurements when measured early after trauma [7]. In the latter study, the combination of increased IL-6 and absence of mHLA-DR recovery was highly predictive of secondary infections (significant odds ratio 18.4). Together, these findings raise the question of how the magnitude of initial severity could dictate the depth of trauma-induced-immunosuppression. A fascinating explanation postulated by Timmerman et al. [1] suggests that DAMPs are a mechanistic explanation.

A second important finding which emerges from this study by Timmermans and colleagues [1] is related to potential pathophysiologic mediators (see Fig. 1). During acute stress, such as that which occurs with ischemiareperfusion injury, trauma and hemorrhage or during chronic pathologies such as cancer or auto-immune disease, cells are dying, and molecules which are normally located in intracellular compartments are released into the extracellular milieu. This modification of the microenvironment can induce a strong inflammatory response without any involvement of a bacterial component $[8,9]$. The molecules secreted are non-immunogenic per se, but upon release and transformation (oxidation, proteolysis) they become immunostimulants and trigger an inflammatory response that can be deleterious to the host [10]. These immunostimulatory molecules released from damaged or dead/dying cells are referred to as DAMPs. To date, a number of

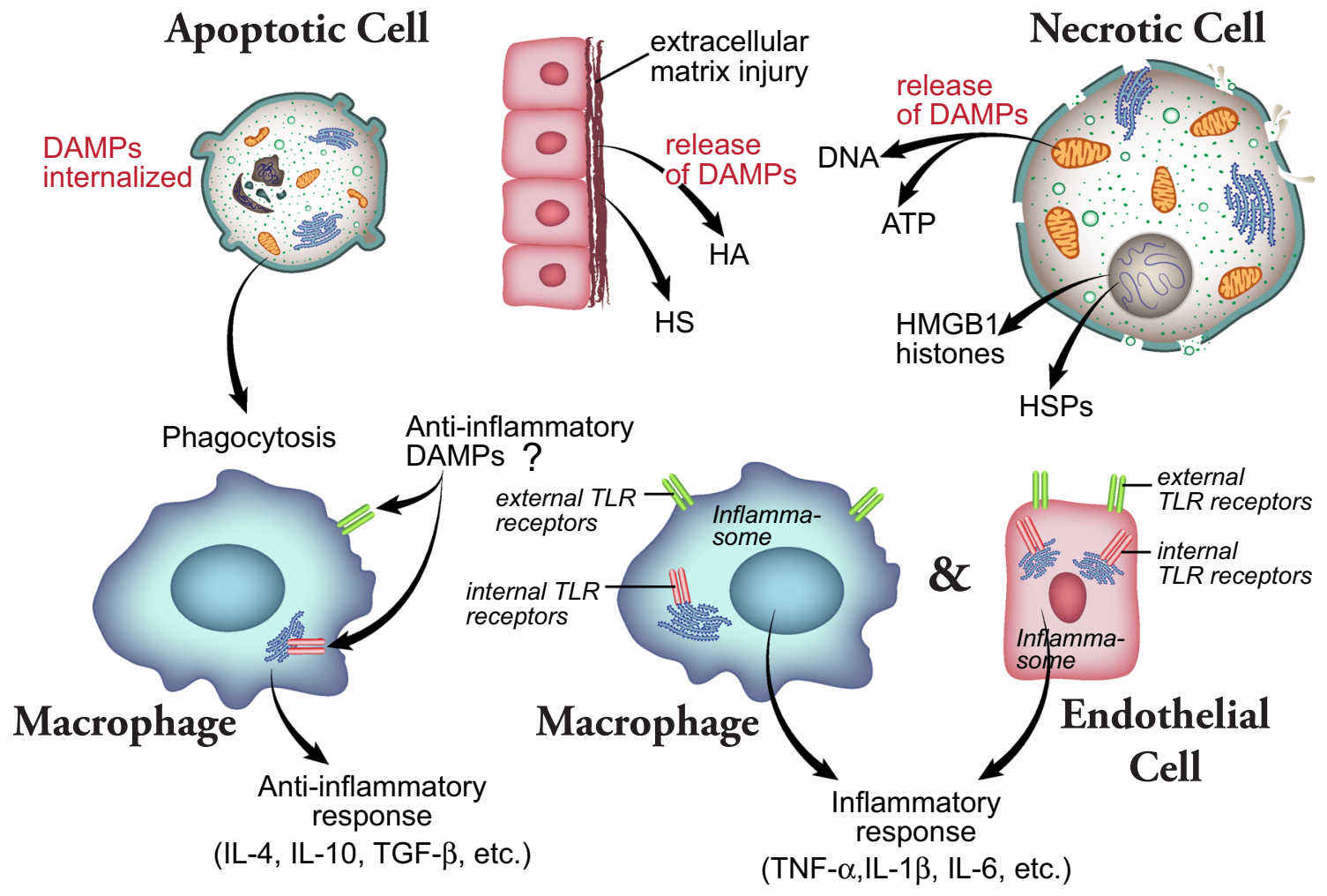

Fig. 1 Danger associated molecular patterns (DAMPs) inducing proor anti-inflammatory responses. In response to diverse internal or external injurious stimuli, DAMPs are released from various intracellular compartments and from the affected extracellular matrix. DAMPs consist of a widely diverse group of molecules, including, extracellularly-derived heparin sulfate $(H S)$ and hyaluronan $(H A)$, and intracellularly-derived high-mobility group box 1 $(H M G B-1)$ proteins, ATP, DNA, and heat shock proteins (HSPs). DAMPs interact with promiscuous pattern recognition receptors, including intracellular and extracellular Toll-like receptors (TLRS) and nucleotide oligomerization domain-like receptors, among others, resulting in formation of the inflammasome. The net result is release of a number of inflammatory cytokines [interleukin $(I L)$, tumor necrosis factor alpha $(T N F-\alpha)$, transforming growth factor beta $(T G F-\beta)]$ which drive inflammation. Recent studies indicate that some DAMPs may lead to an anti-inflammatory cell response. These DAMPs may either act on extracellular or intracellular TLR receptors or be present within apoptotic cells that are consumed by phagocytic cells 
molecules have been identified to function as DAMPs, including heat shock proteins, oxidized lipoproteins, highmobility group box 1 (HMGB-1) proteins, uric acid, ATP, mitochondrial products (mitochondrial DNA), among others. It is fascinating to consider that most of these molecules resemble microbial products called PAMPs (pathogen-associated molecular patterns) and are therefore recognized by the same family of membrane-bound or cytoplasmic pattern recognition receptors, including Tolllike receptors (TLRs) and nucleotide oligomerization domain (NOD)-like receptors [11].

Most of the data available in the current literature show that DAMPs induce a hyper-inflammatory response. However, recent evidence, including that presented by Timmermans et al. [1], suggests that certain DAMPs mayin specific circumstances-induce immunosuppression. As an example, ATP, which is a well-known pro-inflammatory DAMP, may have a paradoxical effect to induce immunosuppression [12]. Indeed, hydrolysis of ATP results in the production of adenosine, a potent inducer of the immunosuppressive cytokine IL-10. These data demonstrate that, depending on the micro-environment in which molecules are released, a particular DAMP could display immunostimulating or immunosuppressing properties. The concentration of extracellular DAMP may be a determinant factor in whether the pro- or anti-immunosuppressive state exists. For example, Trabanelli et al. [13] demonstrated that $250 \mathrm{nM}$ ATP does not modify regulatory $\mathrm{T}$ cells (Treg) function but that $1 \mathrm{mM}$ ATP stimulates suppressive activities and proliferation of Tregs.

Taking into account their immunomodulating properties, DAMPs may represent a potential therapeutic target through the new concept of immunogenic cell death. The term "immunogenic cell death" (ICD) was introduced a few years ago to define a specific type of apoptosis in immunocompetent cells that elicits an immune response against dead cells without the need for adjuvants (see [14] for review). ICD relies on the coordinated release of DAMPs: exposure to an endoplasmic reticulum chaperone on the cell surface, the release of ATP and HMGB1 and the release of cytokine-like type I interferons. These DAMPs recruit and activate antigen-presenting cells, such as dendritic cells, and result in a robust antigen-specific immune response through the activation of $T$ lymphocytes [15]. In the setting of cancer therapy, chemotherapeutics may induce ICD when employed as stand-alone drugs (bleomycin, cyclophosphamide, doxorubicin [16]). Interestingly, other drugs are able to convert nonimmunogenic cell death into ICD (cardiac glycosides, such as digoxin [17]). These drugs could therefore be employed as chemotherapeutic adjuvants in combination with other drugs. Finally, it is important to consider that while DAMPs are danger signals, they are also important players in tissue repair.

These emerging data show that far from univocal functional activities, DAMPS display complex immunomodulating properties, and they may represent a new therapeutic option for both acute and chronic dysregulation of the immune response in several pathologies.

\section{Compliance with ethical standards}

Conflicts of interest All authors declare that they have no conflict of interest to declare.

\section{References}

1. Timmermans K, Kox M, Vaneker M, van den Berg M, John A, van Laarhoven A, van der Hoeven H, Scheffer GJ, Pickkers P (2016) Plasma levels of danger-associated molecular patterns are associated with immune suppression in trauma patients. Intensive Care Med. doi: 10.1007/s00134-015-4205-3

2. Hotchkiss RS, Monneret G, Payen D (2013) Sepsis-induced immunosuppression: from cellular dysfunctions to immunotherapy. Nat Rev Immunol 13:862-874. doi: 10.1038/nri3552

3. Venet F, Lukaszewicz AC, Payen D et al (2013) Monitoring the immune response in sepsis: a rational approach to administration of immunoadjuvant therapies. Curr Opin Immunol 25:477-483. doi: 10.1016/j.coi.2013.05.006
4. Xiao W, Mindrinos MN, Seok J et al (2011) A genomic storm in critically injured humans. J Exp Med. doi: 10.1084/jem.20111354

5. Cheron A, Floccard B, Allaouchiche B et al (2010) Lack of recovery in monocyte human leukocyte antigen-DR expression is independently associated with the development of sepsis after major trauma. Crit Care 14:R208. doi:10.1186/cc9331

6. Gouel-Chéron A, Allaouchiche B, Floccard B et al (2015) Early daily mHLA-DR monitoring predicts forthcoming sepsis in severe trauma patients. Intensive Care Med 41:2229-2230. doi: 10.1007/s00134-015-4045-1
7. Gouel-Chéron A, Allaouchiche B, Guignant C et al (2012) Early interleukin-6 and slope of monocyte human leukocyte antigen-DR: a powerful association to predict the development of sepsis after major trauma. PLoS One 7:e33095. doi: 10.1371/journal.pone.0033095

8. Chan JK, Roth J, Oppenheim JJ et al (2012) Alarmins: awaiting a clinical response. J Clin Invest 122:2711-2719. doi:10.1172/JCI62423DS1

9. Munford RS, Pugin J (2001) Normal responses to injury prevent systemic inflammation and can be immunosuppressive. Am J Respir Crit Care Med 163:316-321. doi: 10.1164/ajrccm.163.2.2007102

10. Venereau E, Ceriotti C, Bianchi ME (2015) DAMPs from cell death to new life. Front Immunol 6:1. doi: 10.1038 /nature 13312 
11. Chen GY, Nuñez G (2010) Sterile inflammation: sensing and reacting to damage. Nat Rev Immunol 10:826-837. doi:10.1038/nri2873

12. Beavis PA, Stagg J, Darcy PK, Smyth MJ (2012) CD73: a potent suppressor of antitumor immune responses. Trends Immunol 33:231-237. doi: 10.1016/j.it.2012.02.009

13. Trabanelli S, Ocadlíková D, Gulinelli S et al (2012) Extracellular ATP exerts opposite effects on activated and regulatory $\mathrm{CD} 4+\mathrm{T}$ cells via purinergic $\mathrm{P} 2$ receptor activation. $\mathrm{J}$ Immunol 189:1303-1310. doi:

10.4049/jimmunol.1103800
14. Kroemer G, Galluzzi L, Kepp O, Zitvogel L (2013) Immunogenic cell death in cancer therapy. Annu Rev Immunol 31:51-72. doi:10.1146/ annurev-immunol-032712-100008

15. Ma Y, Adjemian S, Mattarollo SR et al (2013) Anticancer chemotherapyinduced intratumoral recruitment and differentiation of antigen-presenting cells. Immunity 38:729-741. doi: 10.1016/j.immuni.2013.03.003
16. Casares N, Pequignot MO, Tesniere A et al (2005) Caspase-dependent immunogenicity of doxorubicininduced tumor cell death. J Exp Med 202:1691-1701. doi: 10.1084/jem.20050915

17. Menger L, Vacchelli E, Adjemian S et al (2012) Cardiac glycosides exert anticancer effects by inducing immunogenic cell death. Sci Transl Med 4:143ra99. doi: 10.1126/scitranslmed.3003807 